This is an Accepted Manuscript version of the following article, accepted for publication in Journal of Cross-Cultural Psychology. Biru Zhou, Franca Lacroix, Jun Sasaki, Yunshi Peng, Xia Wang \& Andrew G. Ryder (2014): Unpacking Cultural Variations in Social Anxiety and the Offensive-Type of Taijin Kyofusho Through the Indirect Effects of Intolerance of Uncertainty and Self-Construals, Journal of Cross-Cultural Psychology, 45(10), 1561-1578, DOI: https://doi.org/10.1177\%2F0022022114548483. Copyright (C2014. It is deposited under the terms of the Creative Commons

Attribution-NonCommercial-License (http://creativecommons.org/licenses/by-nc/4.0/), which permits non-commercial re-use, distribution, and reproduction in any medium, provided the original work is properly cited.

\title{
Unpacking Cultural Variations in Social Anxiety and the Offensive-Type of Taijin Kyofusho Through the Indirect Effects of Intolerance of Uncertainty and Self-Construals
}

\author{
${ }^{1}$ Biru Zhou, ${ }^{1}$ Franca Lacroix, ${ }^{2}$ Jun Sasaki, ${ }^{3}$ Yunshi Peng, ${ }^{3}$ Xia Wang, and ${ }^{1, \text { and }}{ }^{4}$ Andrew G. Ryder \\ ${ }^{1}$ Concordia University \\ ${ }^{2}$ Osaka University \\ ${ }^{3}$ Hunan Normal University \\ ${ }^{4}$ Jewish General Hospital
}

\begin{abstract}
This paper presents two studies that aim to unpack cultural variations in general social anxiety and the offensive-type of Taijin Kyofusho (OTKS) - a type of social anxiety characterized by the extreme fear of offending others. Cultural variations in the expression and manifestation of social anxiety are well established; however, the mechanisms underpinning this relation are unclear. The present studies use the Parallel Multiple Mediation Model to study how social anxiety and OTKS are jointly shaped by self-construal and intolerance of uncertainty. Study 1 compared Euro-Canadians and Chinese migrants in Canada. Results showed a mean group difference in OTKS, but not social anxiety, with the difference mediated by intolerance of uncertainty (IU). Study 2 tested this pattern of multiple mediations in Japanese, Chinese and Euro-Canadian cultural contexts. Results showed significant differences among these three cultural groups on both social anxiety and OTKS via multiple mediators (e.g., independent vs. interdependent self-construals and IU). Findings in both studies revealed that OTKS seems to be a psychopathology that is not specific to Japanese participants. The underlying mechanisms and processes of OTKS are also significantly different from social anxiety. Significant cultural variations in social anxiety and OTKS between Chinese vs. Japanese cultural contexts were observed in Study 2. These studies demonstrate the conceptual and empirical advantages of using more complex models to unpack the psychological mechanisms shaping cultural variations in social anxiety and OTKS.
\end{abstract}

Keywords: Social Anxiety, Offensive-type TKS, Multiple Mediation, Intolerance of Uncertainty

Anxiety is an ubiquitous human experience and pervasive anxiety during social situations, to the point of significant impairment or distress, is understood within most societies worldwide as problematic (Good \& Kleinman, 1985). Nonetheless, cultural variations exist both in the expression of symptoms and in the social context where these anxiety symptoms are elicited (Dinnel, Kleinknecht, \& TanakaMatsumi, 2002; Kleinknecht, Dinnel, Kleinknecht, Hiruma, \& Harada, 1997; Kirmayer, 1991). There is a growing literature detailing the ways in which culture shapes social anxiety

Corresponding Author:

Biru Zhou, Concordia University, PY-146, 7141 Sherbrooke West, Montreal, Quebec, Canada H4B 1R6. Email:

b_zho@live.concordia.ca
(SA), with a particular emphasis on East Asian cultural contexts. SA disorder, according to the fifth edition of the Diagnostic and Statistical Manual of Mental Disorders (DSM-5; American Psychiatric Association, 2013), is marked by the persistent fear of social or performance situations in which embarrassment may occur. In contrast, SA in Japan and in China is understood differently. Taijin Kyofusho (TKS) is loosely translated as "anthropophobia" or "phobia of interpersonal relations" (Ono et al., 2001). Patients who suffer from this syndrome report experiencing a variety of symptoms, such as fears of eye-to-eye contact, blushing, displaying improper facial expressions in the presence of others, looking at others, or body odour being noticed (e.g., Takahashi, 1989).

Furthermore, elevated levels of self-reported SA are generally found in East Asian student samples relative to their Euro-American counterparts (e.g., Dinnel et al., 2002; Hong \& Woody, 2007; Okazaki, Liu, Longworth, \& Minn, 2002). 
Studies related on SA and TKS also demonstrated that people in East Asian cultural contexts are more likely to report fears of offending others in social situations whereas people in Euro-American or Euro-Canadian cultural contexts are more likely to report social fears that are self-oriented (e.g., Dinnel et al., 2002; Kleinknecht et al., 1997). Recently, the "Western" defined SA disorder included the fear of offending others as part of the diagnostic criteria in DSM-5 (American Psychiatric Association, 2013). Most studies examining SA and TKS, however, are limited to documenting group differences, and potential explanations are relegated to the discussion section rather than being built into the methodology. As a result, we have little data about why these cultural variations are observed. A notable recent exception is a study of Japanese and American university students by Norasakkunkit, Kitayama, and Uchida (2012), which examined the role of holistic cognition as the basic cognitive feature distinguishing self-oriented vs. other-oriented social anxiety.

The primary goal of the two studies presented in this paper is to unpack both cultural and individual variations in social anxiety and the offensive-type of Taijin Kyofusho for a better understanding of how "culture matters". By using both Chinese and Japanese samples, the second study in particular moves away from the common assumption that East Asian cultural groups are interchangeable. We will begin by considering cultural variations in self-construals, and then move to the potential role of intolerance of uncertainty as mechanisms operating in social anxiety and the offensive-type of Taijin Kyofusho.

\section{Independent vs. Interdependent Self-Construals}

The cultural variations in social anxiety due to a different understanding of oneself in relation to others have been well documented in previous research (e.g., Dinnel et al., 2002; Kirmayer, 1991). The important distinction between independent and interdependent self-construals has been shown to relate to social anxiety in different cultural settings (Dinnel et al., 2002; Hong \& Woody, 2007; Okazaki et al., 2002).

Any particular person may react in a more independent or interdependent way depending on the specific situation. These two ways of understanding the self are not mutually exclusive, even though one might be more likely to be activated than the other in a given context. The culturally defined views of the self (independent vs. interdependent) may produce different behaviours in response to social anxiety (Dinnel et al., 2002; Markus \& Kitayama, 2010). In North American cultural contexts where the independent self-construal predominates, social anxiety problems tend to emerge from the fear of embarrassing oneself by acting inappropriately. In contrast, in Japanese or Chinese cultural contexts, social anxiety problems tend to emerge from the fear that inappropriate social interactions might offend others. In other words, for North American populations, the underlying fear of so- cial anxiety is more "self" oriented, whereas the underlying fear that is most prevalent among East Asian populations tends to be more "other" oriented (Choy, Schneier, Heimberg, Oh, \& Liebowitz, 2008; Kasahara, 1974; Kirmayer, 1991; Kleinknecht et al., 1997).

\section{The Offensive-Type of Taijin Kyofusho}

Many of the culturally distinctive aspects of TKS are combined in a subtype called the offensive-type of TKS (OTKS). Patients with OTKS fear that perceived physical defects or inappropriate behaviours might disturb, offend, or even harm others (Choy et al., 2008; Kasahara, 1987). For instance, a patient may be reluctant to look into another person's eyes because he/she fears that his/her eye gaze would make other people unbearably uncomfortable or might even hurt others. Although there is a general consensus that TKS resembles SA, patients with OTKS can be distinguished from patients with DSM-defined SA disorder by their persistent concerns that their symptoms might disturb others (Iwase et al., 2000; Kasahara, 1987; Nagata et al., 2006; Ono et al., 2001). In other words, OTKS sufferers tend to focus on the impact of their symptoms on other people while SA disorder patients in general tend to focus on the impact of the symptoms on themselves.

Studies have provided some support for the differing effects of self-construals on SA and TKS (Dinnel et al., 2002; Kleinknecht et al., 1997; Norasakkunkit et al., 2012). Kleinknecht and colleagues conducted a series of studies examining the relation between self-construals and general TKS (not OTKS specifically) among American and Japanese university students. Results indicated that participants who construed themselves as less independent were more likely to report higher levels of TKS in both Japanese and American samples. The relation between interdependent self-construal and TKS was either small or nonsignificant in both samples. However, cultural context, namely Japanese vs. American, accounted for additional variability in TKS symptoms, with Japanese participants scoring higher. Clinical research, meanwhile, has shown that the TKS-specific fear of offending others or making others uncomfortable could be identified among North American patients with European heritage (e.g., Choy et al., 2008; Clarvit, Schneier, \& Liebowitz, 1996; Rector, Kocovski, \& Ryder, 2006). In addition, the occurrence of TKS symptoms is not strongly related to interdependent self-construal among Japanese students (Dinnel et al., 2002; Kleinknecht et al., 1997).

Self-construals (i.e., independent vs. interdependent selfconstruals) alone do not fully account for cultural variations in SA and TKS (e.g., Dinnel et al., 2002). Indeed, studies of cultural variation in independent vs. interdependent selfconstruals have often yielded mixed results (Kashima, Koval, \& Kashima, 2011; Norasakkunkit et al., 2012; Matsumoto, 2002). The relation between culture and the self is highly 
complex -not only do they have a relation of mutual constitution (Markus \& Kitayama, 2010), they also change over time (Kashima et al., 2011). Individual people within a cultural context differ from one another, and they can endorse, partially endorse, reject, or subvert cultural meanings and practices in innumerable ways (Ryder, Ban, \& ChentsovaDutton, 2011). We cannot simply claim that people living in East Asian cultural contexts are always more interdependent cross different situations than people living in "Western" cultural contexts. It is important to continue the investigation of culturally-shaped self-construals in order to understand the mechanisms that contribute to the variations observed between East Asian vs. "Western" cultural contexts in SA and OTKS.

\section{Intolerance of Uncertainty}

Dugas, Schwartz, and Francis (2004) have defined Intolerance of Uncertainty (IU) as a person's excessive tendency not to tolerate a negative event may occur, however low the probability. People high in IU find the many ambiguous aspects of everyday life to be intolerable and stressful. IU was originally believed to contribute uniquely to worry and generalized anxiety disorder (e.g., Dugas et al., 2004). Recently, researchers found evidence that IU is also related to social anxiety (Boelen \& Reijntjes, 2009; Carleton, Collimore, \& Asmundson, 2010; Carleton et al., 2012; McEvoy \& Mahoney, 2012), such that the effect of IU has unique contributions to social anxiety over and above fear of negative evaluation. Given that socially anxious persons fear that they are constantly under scrutiny by others (Hofmann, Asnaani, \& Hinton, 2010), it is not surprising that social situations are intolerable for them because these situations are full of uncertainties -not least, the potential to be negatively evaluated (Boelen \& Reijntjes, 2009).

IU at the country level has been measured by uncertainty avoidance, and research has linked higher levels of this cultural value with a higher prevalence of anxiety (Hofstede, 1984, 2001). Heinrichs et al. (2006) tried to link social norms with social anxiety, and their studies provided evidence that obvious and elaborated social rules for appropriate behaviours are indeed associated with higher SA. Both Heinrichs et al.'s and Hofstede's studies indicate that cultural rules and norms play nonnegligible roles in individuals' SA and well-being.

As mentioned previously, there are significant cultural variations in the expressions of SA, (e.g., OTKS vs. DSMdefined SA). These variations may be partially explained by independent vs. interdependent self-construals (e.g., Dinnel et al., 2002; Kleinknecht et al., 1997; Okazaki, 1997; Norasakkunkit et al., 2012). However, cultural variations in IU as it relates to SA and OTKS at the individual level have yet to be examined. IU is considered as an important transdiagnostic maintenance factor for various anxiety disorders and depression (McEvoy \& Mahoney, 2012). In an effort to test whether IU has the same effect on OTKS as on SA, this paper aims to unpack cultural variations in SA and OTKS by exploring multiple mediation effects (both direct and indirect effects) of self-construals and IU. We attempt to go beyond comparison of group differences and strive to identify the mechanisms underpinning these cultural variations in SA and OTKS.

First, this project will try to replicate previously identified cultural variations in SA and OTKS through the indirect effects of self-construals. Secondly, we will investigate the additional contribution made by the indirect effect of IU on social anxiety and OTKS. This paper presents two studies. Hong and Woody (2007) tested the mediating effects of self-construals in their paper comparing Korean with EuroCanadian participants. Study 1 extends Hong and Woody's examination to the Chinese cultural context. Furthermore, the two self-construals were entered separately in two different models in Hong and Woody's study. Since independent and interdependent self-construals are not mutually exclusive (Markus \& Kitayama, 2010), it is important to enter them in the multiple mediation model simultaneously in order to obtain the unique indirect effect of each mediator after controlling for the effects of the other mediators. Study 1 will examine these three mediators simultaneously using the Parallel Multiple Mediation Model (defined in the next section; Hayes, 2013) among Euro-Canadians and Chinese migrants who are currently residing in Montréal, Québec, Canada. Study 2 will examine the same variables through cross-group comparisons (Japanese in Japan vs. Chinese in mainland China vs. Euro-Canadians in Canada).

\section{Study 1}

OTKS is often studied among Japanese and Korean populations (e.g., Choy et al., 2008; Kasahara, 1987; Kleinknecht et al., 1997; Kim, Rapee, \& Gaston, 2008), but it is rarely examined among Chinese populations. Not to presume all Asian cultural groups are interchangeable, a multiple mediation analysis ${ }^{1}$ was used to examine cultural variations (EuroCanadians vs. Chinese migrants) in social anxiety and OTKS via the operating mechanisms of independent self-construal, interdependent self-construal and IU (see conceptual models in Figure 1). In our case, we estimated the direct effects (denoted as $c^{\prime}$ ) of cultural variation in social anxiety and OTKS, and the indirect effects of self-construals and IU. Specifically, we predicted that the contrast between Euro-Canadians and Chinese migrants on both social anxiety and OTKS would

\footnotetext{
${ }^{1}$ Multiple mediation models discussed in this paper were conducted using an open source SPSS macro "PROCESS" available at http://www.afhayes.com/. The three cultural group comparisons in Study 2 were made possible by the procedures outlined by Hayes and Preacher (in press) for analyzing multicategorical independent variables.
} 
be explained at least in part by the indirect effects of selfconstruals and IU.

\section{Method}

\section{Participants}

Participants in Study 1 were drawn from two sources. Approximately $26 \%$ of the participants were originally recruited from a larger project requiring them to participate in pairs with a friend. One member of each pair was randomly excluded in this study in order to maintain independence of observations. The rest of the participants were independently recruited via the university's participant pool. Data for seven participants were dropped because they skipped at least one questionnaire. Our final analyses were based on a sample of 376 participants, almost all of whom (97\%) were university students from the Montréal area.

The Euro-Canadian participants (215 females, 52 males, mean age $=22.43, S D=4.80$ ) were all born in Canada and self-identified as (a) having European heritage and (b) being "White (Caucasian)". The Chinese heritage participants (60 females, 49 males, mean age $=21.46, S D=4.70$ ) were all born in China and left China after the age of 8 . The Chinese sample in Study 1 consisted of either international students or immigrants who were residing in the Montréal area at the time of the study.

\section{Measures}

The Self-Construal Scale (SCS; Singelis, 1994) is a 30item scale that measures independent self-construal and interdependent self-construal using a 7-point rating scale (ranging from $1=$ Completely Disagree to $7=$ Completely Agree). SCS consists of two subscales: independent selfconstrual and interdependent self-construal.

The Intolerance of Uncertainty Scale (IUS; Buhr \& Dugas, 2002) is a 27-item scale that are related to uncertainty, one's reactions to ambiguous situations, and the cognitive and emotional implications of being uncertain. It has a 5-point Likert scale ranging from 1 (Not at all characteristic of me) to 5 (Extremely characteristic of me). An example of IUS is: "Uncertainty makes life intolerable."

The Social Interaction Anxiety Scale (SIAS; Mattick \& Clarke, 1998) is a 20-item widely used self-report that measures social anxiety. It is designed to assess social anxiety as typically defined by "Western" standards during interactions with others. It has a 5-point rating scale ranging from 0 (Not at all characteristic or true of me) to 4 (Extremely characteristic or true of me).

The Taijin Kyofusho Questionnaire (TKSQ; Choy et al., 2008 ) is a 30-item self-report that measures the severity of three sets of fears associated with ten physical and/or behavioral symptoms that are characteristic of TKS and social anxiety. Participants were asked to rate their fears associated with "embarrassing yourself", "making someone uncomfortable", and "offending someone" for each symptom using a 4-point rating scale ranging from 1 (I would not be fearful at all) to 4 (I would be extremely fearful). Since our study focused on only the offensive-type of TKS (OTKS), we only used the 20 items that were related to the fear of "making someone uncomfortable" and "offending someone". According to the conceptualization of OTKS (e.g., Kasahara, 1974; Kirmayer, 1991), the fear of embarrassing others (e.g.,family or friends) should be the most prominent fear underlying OTKS, whereas embarrassing oneself should be the underlying fear for "Western" social anxiety. Therefore, the 10 items measuring embarrassment towards oneself were not included in the analyses.

The Language Questionnaire consists of three language related questions. Chinese participants were asked to rate their level of proficiency in speaking, understanding and writing in English using a 7-point rating scale with (1) = Very poor to $(7)=$ Native-like. The mean level of English proficiency was $4.89, S D=1.06$. No ESL class student was recruited for this project. The recruitment criteria, verified by the language questionnaire, indicated that Chinese participants in this study have adequate English proficiency to understand the questionnaires.

All scales' internal consistencies reached satisfactory levels in the same range as estimates reported in the existing literature. Cronbach's alpha coefficients are presented in Table 1 . Table 2 contains the correlations among all variables by cultural groups.

\section{Procedure}

All participants completed the online questionnaires hosted on a secured server. There was no time limit for completing the online questionnaire, allowing participants to complete it at their preferred pace. Participants were informed at the beginning of the survey that the study was completely voluntary, that they could discontinue the study at any time, and that they did not have to respond to questions that they did not want to answer. Upon completion of the survey, all participants had the choice between obtaining \$20 in cash or psychology department participant pool credits if they were eligible. A debriefing information page appeared at the end of the survey.

\section{Results}

\section{Preliminary Analysis}

About $0.3 \%$ of responses were missing from our data set. Little's Missing Completely At Random (MCAR) test was conducted for the Euro-Canadian participants and Chinese participants separately. Results showed that for both groups, responses were missing completely at random, $\chi^{2}(5057)=5158.12, p=.16$ for Euro-Canadians and 
$\chi^{2}(2146)=2036.11, p=.96$ for Chinese participants. Therefore, Maximum-Likelihood data imputations were employed for both cultural groups separately using the ExpectationMaximization algorithm (McKnight, McKnight, Sidani, \& José, 2007). Mean scores for each scale and subscale were then calculated. Univariate outliers were winsorized and no multivariate outliers were observed using Mahalanobis Distances. The homogeneity of variance assumption was not met for some measures, with unequal variances between European-Canadian and Chinese participants for SIAS, $F(1$, $374)=10.25, p=.001$, and OTKSQ, $F(1,374)=116.53, p$ $<.001$. We, therefore, used the heteroscedasticity-consistent standard error estimator within the "PROCESS" macro to correct for this issue (Hayes \& Cai, 2007; Hayes, 2013).

Tests for homogeneity of regression slopes (Hayes \& Preacher, in press) using ANCOVA showed an interaction between cultural groups and interdependent self-construal for SIAS, $F(1,368)=9.70, p=.002$. As a result, interdependent self-construal was not entered in further analyses involving SIAS because homogeneity of regression slopes could not be assumed. As one-way ANOVA showed that age differed between the two cultural groups, $F(1,374)=$ $14.07, p<.001$, it was entered as a covariate in the multiple mediation analyses.

\section{Multiple Mediation Analysis}

In this section, we examined cultural variations on social anxiety through the effects of self-construals (e.g., Hong \& Woody, 2007) and IU, controlling for recruitment methods and age. Cultural group (i.e., European-Canadians vs. Chinese) was the independent variable and the outcome variables were social anxiety as measured by SIAS and the offensive type of TKS as measured by OTKSQ. The two mediators - independent self-construal and IU - were entered at the same time in the multiple mediation analysis for SIAS. Independent and interdependent self-construals, and IU, were entered as mediators for the analysis of OTKSQ. All indirect effects reported below were unique effects specific to the particular mediator, controlling for all other effects. All direct effects (noted as $c^{\prime}$ ) were also the unique effects between the independent variable and the outcome variable, after taking into account all other effects in the model. The regression coefficient between cultural groups and the mediator was denoted as a, whereas the regression coefficient between the mediator and the outcome variable was denoted as b. Since there were pairs of analyses (i.e., SIAS vs. OTKSQ) involving the same variables, we set significant $p$-value to .025 using Bonferroni correction in order to avoid the risk of inflating Type I error.

SIAS. The mediation analysis for SIAS indicated that the direct effect of cultural groups on SIAS was marginally significant after accounting for the indirect effects of independent self-construal and IU, $c^{c}=.16, p=.03$, biascorrected $97.5 \%$ CI [-.004, .32], with Chinese participants scoring higher on SIAS (see Table 1). The EuropeanCanadian and Chinese groups differed on SIAS due to the indirect effects of independent self-construal (point estimate: .09, bias-corrected $97.5 \%$ CI $[.05, .16])$ and IU (point estimate: .29, bias-corrected $97.5 \%$ CI [.20, .41]). The mediation analysis suggested that the group differences between Euro-Canadians and Chinese migrants on SIAS were transmitted through the indirect effect of independent self-construal and IU. Chinese migrants reported lower levels of independent self-construal, compared to the EuroCanadians $(a=-.40, p<.001)$ and these lower levels of independent self-construal were then related to higher levels of social anxiety measured by SIAS $(b=-.24, p<.001)$. However, for IU, Chinese migrants reported higher scores on IU ( $a=.73, p<.001)$, which was associated with higher scores on SIAS $(b=.40, p<.001)$.

OTKSQ. A different pattern of results emerged from the multiple mediation analysis for OTKSQ. The direct effect of cultural group on OTKSQ remained after accounting for the indirect effects of independent self-construal, interdependent self-construal and IU $\left(c^{c}=.47, p<.001\right.$, biascorrected $97.5 \%$ CI $[.31, .63])$, with Chinese participants reporting higher OTKSQ scores (see Table 1). In contrast to the SIAS model, there was no indirect effect of independent self-construal (point estimate: .02, bias-corrected $97.5 \% \mathrm{CI}$ $[-.01, .06])$ or interdependent self-construal (point estimate: .01 , bias-corrected $97.5 \%$ CI $[-.002, .04]$ ) for OTKSQ, although the indirect effect of IU remained, point estimate: .17 , bias-corrected $97.5 \%$ CI [.09, .26]. Group differences on OTKSQ via the influence of IU indicated that Chinese migrants reported higher levels of IU $(a=.73, p<.001)$ and these higher levels of IU were then associated with higher levels of OTKS $(b=.23, p<.001)$.

\section{Discussion}

Mean differences between European-Canadians and Chinese migrants were observed for social anxiety (marginally) and OTKS with the latter group reporting higher on both scales, after controlling for all the indirect effects. Group differences for SIAS could be explained through the indirect effects of independent self-construal and IU, whereas for OTKS, the group differences could be explained through the indirect effect of IU only. The effect of independent selfconstrual continued to predict the "self-oriented" SA, but not the "other-oriented" OTKS. On the other hand, IU appeared to be consistently predictive of both SIAS and OTKS in Study 1.

The Chinese sample in this study included both immigrants and international students. We did not have information regarding the Chinese migrants' migration history nor 
did we control for the years they had been in Canada. In comparison, our Euro-Canadian participants were all born and raised in Canada. It is possible that some other variables, such as acculturation attitudes and networks of social interactions among Chinese migrants, could account for the results we have observed. In order to obtain cross-group comparisons that are not tainted by acculturation attitudes or other migration factors, we examined the same multiple mediation models in three cultural contexts (i.e., Canada, Japan and mainland China) in the next study.

\section{Study 2}

Chinese and Japanese cultural contexts tend to foster a more well developed interdependent self-construal, meaning that connectedness with others is particularly salient in Chinese and Japanese cultural contexts (Markus \& Kitayama, 2010). Substantial variations exist between these contexts regarding the appropriateness and the importance of connecting with others. Hendry (2003) and Nakane (1970) described Japanese society as highly structured, particularly regarding interpersonal relationships. A highly elaborated system of proper social behaviour -"good manners" -are embedded within a complex hierarchical ranking system (Nakane, 1970; Sugimoto, 2009). There are many differences of rank based on relative age, year of entry or years of service. Ranking order is central to fixing social order and maintaining social cohesion in Japanese cultural contexts (Hendry, 2003). Since this highly structured social system is essential for harmonious interpersonal relations, uncertainty during social interactions may be particularly difficult to tolerate. IU may therefore be an important antecedent of SA and OTKS in Japanese cultural contexts.

In contrast, despite the strong focus on interdependence, the hierarchical ranking system in China is much less stringent than in Japan partially due to political efforts to abolish "class oppression" since the mid-20th century (Louie, $2008)^{2}$. For instance, regular workers do not need to bow to greet the president of a company in China, but it would be considered extremely rude and disrespectful not to do so in Japan. The societal variations in the hierarchical ranking system are illustrated by a communication study about politeness in Japan and China (Lin, 2013). When participants were asked what came to mind when they heard the word "politeness", more than twice as many Japanese participants than Chinese participants associated "politeness" with senior and junior positions or hierarchical relationships.

Furthermore, many studies had examined OTKS among Japanese and Korean samples (e.g., Kim et al., 2008; Ono et al., 2001). OTKS is considered as a "other-oriented" fear that was first identified in Japan (Ono et al., 2001; Kasahara, 1974, 1987). Conceptually, lower independent selfconstrual and/or higher interdependent self-construal should be related to higher levels of OTKS. However, according to Hofstede (2001) and Hofstede, Hofstede, and Minkov (2010), Japan scored higher on the individualism dimension than China. Japanese cultural contexts tend to be more collectivistic in the Western standards and more individualistic in the Asian standards. Since the comparison between Japanese and Chinese cultural contexts in SA and OTKS is rarely made, it is unclear whether there are cultural variations in self-construals between these two cultural contexts. If there are such variations, it is also unclear whether and how these variations will contribute to the "self-oriented" SA and the "other-oriented" OTKS. Therefore, three cultural group comparisons among Euro-Canadians, Japanese and Chinese participants in Study 2 will help to address these issues.

We hypothesized that there would be mean differences between Chinese and Japanese participants in the indirect effects of self-construals and IU. These mean differences would also be displayed in the comparisons between EuroCanadians vs. Chinese, and between Euro-Canadians vs. Japanese. Due to the rich and elaborated system of proper social manners in Japanese society, we anticipated that the indirect effect of IU on social anxiety and OTKS would be stronger for Japanese participants, compared to Chinese and Euro-Canadian participants.

\section{Method}

\section{Participants}

Participants were Japanese, Chinese and Euro-Canadian university students. Euro-Canadian participants (169 females, 29 males, mean age $=21.81, S D=4.30$ ) were all born in Canada and self-identified as (a) having European heritage and (b) being "White (Caucasian)". Chinese participants (52 females, 75 males, mean age $=19.71, S D=.94$ ) were all born in China and were attending university in Changsha, Hunan Province, China at the time of the study. Japanese participants ( 68 females, 53 males, mean age $=21.03, S D=$ 4.60) were all born in Japan and were attending university in either the Kanto or the Chubu region, Japan.

\section{Measures}

The same scales as in Study 1 were used in this study. See Table 3 for correlations among variables. All scales were translated into Japanese and Chinese by three bilingual researchers who have background in psychology working on this project using a modified version of TRAPD team translation model (Harkness, 2003). The researchers formed two

\footnotetext{
${ }^{2}$ Even though there is no direct comparison between Chinese and Japanese hierarchical ranking systems per se, the historical and political accounts of modern Chinese development have provided evidence that social hierarchy still exists in China but it is not as heavily embedded in daily social interactions as in Japan and it is not as visible as in Japanese social etiquette (Alon, 2003; Hendry, 2003; Louie, 2008; Sugimoto, 2009
} 
committees of translation: one for Japanese and one for Chinese. The three translators first divided the work of translating scales into the other languages. Then, translators reviewed each other's translation work. Reviewers examined all scales item by item again in depth and identified "problematic" items. Then, all translators/reviewers discussed all items in group meetings, facilitated by telephone or VOIP services for international communications, as many times as needed until they reached an agreement on the translation. The authors in this paper served as adjudicators to help translators/reviewers to resolve any translation related problems during the process.

Finally, translators read though all scales independently again to check for typos and any other mistakes. If concerns of certain items arose during this stage, translators/reviewers would go back to review and discuss those items again until an agreement was reached. Adjudicators would then decide whether the modifications were ready to be finalized. A final version of the questionnaires were then discussed in conference meetings by translators/reviewers and adjudicators. All scales' internal consistencies reached satisfactory levels in the same range as estimated reported in the existing literature (see Table 4).

\section{Procedures}

Euro-Canadian participants completed the online questionnaires hosted on a secured server as in Study 1. EuroCanadian participants received psychology department participant pool credits for their participation. A debriefing information page was presented at the end of the survey. For Japanese and Chinese participants, all questionnaires were completed in class using paper and pencil as part of their class participation. All participants were informed at the beginning of the survey that this study was completely voluntary, that they could discontinue the study at any time and that they did not have to respond to questions that they did not want to answer.

\section{Results}

\section{Preliminary Analysis}

About $0.5 \%$ of responses were missing from our data. Little's MCAR test was conducted for the Euro-Canadian, Japanese and Chinese participants separately. The results showed that for all three groups, the missing responses were missing completely at random, $\chi^{2}(4727)=4856.58, p=$ .09 for Canadians, $\chi^{2}(4329)=4207.24, p=.91$ for Chinese, and $\chi^{2}(2293)=2189.05, p=.94$ for Japanese participants. Therefore, Maximum-Likelihood data imputations were employed for the three cultural groups separately using the Expectation-Maximization algorithm (McKnight et al., 2007). Mean scores for each scale and subscale were then calculated.
Univariate outliers were winsorized and no multivariate outlier using Mahalanobis Distances was observed in this study. A one-way ANOVA showed that there were age differences among the three groups, $F(2,446)=11.96, p<.001$. Age was entered as a covariate in the following analysis. Homogeneity of regression slopes using ANCOVA showed that the interaction between cultural groups and independent self-construal was significant, $F(2,437)=5.83, p=.003$ for SIAS. As a result, independent self-construal was not entered in the analysis predicting SIAS (Hayes \& Preacher, in press).

The homogeneity of variance was not met for some measures, with unequal variances among the participants from the three cultural groups for SIAS, $F(2,446)=18.07, p<$ .001 , and in OTKSQ, $F(2,446)=9.63, p<.001$. We therefore used the heteroscedasticity-consistent standard error estimator to correct for this issue (Hayes \& Cai, 2007; Hayes, 2013).

\section{Multiple Mediation Analysis}

In order to avoid inflating Type I error with multiple analyses using the same outcome variables, we set the significant $p$ value to .01. Multiple mediation models using cultural groups as the independent variable, and SIAS and OTKSQ as the dependent variables were analyzed in Study 2, see Figure 1 for the conceptual model.

Euro-Canadians vs. Chinese. The direct effect of cultural group mean differences on SIAS was significant $(c$ $=-.30, p<.001$, bias-corrected 99\% CI $[-.48,-.12])$, with Euro-Canadian participants rating higher (see Table $4)$. Chinese participants rated higher on interdependent selfconstrual than Euro-Canadian participants, $a=.31, p<.001$. There was no mean difference between these two groups for $\mathrm{IU}, a=.06, p=.36$. There was no indirect effect found for the prediction of SIAS.

For the analysis of OTKSQ, there was no direct effect of cultural group mean differences $\left(c^{\star}=-.05, p=.41\right.$, biascorrected $99 \%$ CI $[-.21, .11])$, nor was there an indirect effect for IU, point estimate: .02, bias-corrected 99\% CI [-.03, .08]. There were, however, indirect effects of interdependent self-construal on OTKSQ (point estimate: .07, bias-corrected $99 \%$ CI $[.02, .14])$ and independent self-construal (point estimate: .04, bias-corrected $99 \%$ CI [.002, .09]) in predicting OTKSQ.

Chinese participants rated higher on interdependent selfconstrual than Euro-Canadians, $a=.31, p<.001$. Higher interdependent self-construal was related to higher OTKSQ, $b=.23, p<.001$. The mean difference of OTKSQ between Euro-Canadian and Chinese participants, with Chinese participants rating higher (see Table 4), was accounted for partially by the indirect effect of interdependent self-construal. Euro-Canadians rated marginally higher on independent selfconstrual than Chinese participants, $a=-.21, p=.01$. Higher independent self-construal was negatively related to OTKSQ, 
$b=-.18, p<.001$. The mean difference of OTKSQ between these two groups can also be explained by the indirect effect of independent self-construal.

Euro-Canadians vs. Japanese. There was no direct effect of cultural groups on SIAS, $c^{c}=.03, p=.77$, biascorrected $99 \%$ CI $[-.19, .23]$. There was no group difference on interdependent self-construal, $a=-.12, p=.09$. However, Japanese participants reported higher levels of IU compared to the Euro-Canadian participants, $a=.63, p<.001$. As predicted, there was an indirect effect of cultural groups on SIAS via IU, point estimate: .35, bias-corrected 99\% CI from .22 to .50. Japanese participants reported higher scores on IU, and then IU was related to higher levels of SIAS, $b=$ $.55, p<.001$. There was no indirect effect of interdependent self-construal for predicting SIAS.

Turning to the prediction of OTKSQ, there was no direct effect of cultural groups on OTKSQ, $c=.05, p=.52$, bias-corrected $99 \%$ CI $[-.15, .25]$, but there were indirect effects of independent self-construal (point estimate: .05, bias-corrected $99 \%$ CI $[.02, .11]$ ) and IU, point estimate: .15 , bias-corrected $99 \%$ CI $[.08, .25]$. Euro-Canadians reported higher levels of independent self-construal than did Japanese participants $(a=-.29, p<.001)$, and higher independent self-construal was related to lower OTKSQ, $b=$ $-.18, p<.001$. The group mean differences between EuroCanadians and Japanese were mediated by the effect of independent self-construal. Japanese participants also reported higher levels of IU $(a=.63, p<.001)$, which in turn predicted higher levels of OTKSQ, $b=.24, p<.001$. In short, IU was a significant mediator operating for the mean differences between Euro-Canadians and Japanese on both social anxiety and OTKS. Specifically, Japanese participants endorsed higher levels of IU and then IU was positively related to both SIAS and OTKSQ.

Chinese vs. Japanese. There was a mean difference on SIAS between Chinese and Japanese participants $\left(c^{\circ}=.32\right.$, $p<.001$, bias-corrected 99\% CI [.11, .54]), with Japanese participants scored higher (see Table 4). There were also group mean differences on interdependent self-construal ( $a$ $=-.43, p<.001)$ with Japanese participants scoring lower than Chinese participants did. The only indirect effect for SIAS involved IU, point estimate: .31, bias-corrected $99 \%$ CI $[.19, .45]$. Japanese participants reported higher levels of IU than Chinese participants $(a=.56, p<.001)$, and IU was positively related to SIAS, $b=.55, p<.001$.

For the analysis of OTKSQ, there was no direct effect of cultural groups, $c=.10, p=.22$, bias-corrected $99 \% \mathrm{CI}$ $[-.11, .31]$. There were indirect effects for both interdependent self-construal (point estimate: -.10, bias-corrected 99\% CI $[-.18,-.04])$ and IU, point estimate: .13, bias-corrected 99\% CI [.07, .22]. Japanese participants reported lower levels of interdependent self-construal and higher levels of IU (see Table 4). Interdependent self-construal and IU were then both positively related to OTKSQ, $b=.23$ and $b=.24$ respectively, $p s<.001$.

\section{General Discussion}

It has been well documented that there are cultural variations in psychopathology (Dinnel et al., 2002; Heinrichs et al., 2006; Kirmayer, 1991; Takahashi, 1989). However, it is still an ongoing task to examine the underlying processes of these variations (Draguns \& Tanaka-Matsumi, 2003). This paper is the first to our knowledge to consider multiple cognitive pathways (i.e., self-construals and IU) to unpack the cultural influences on social anxiety and OTKS. This approach represents one way to respond to the preference in culturalclinical psychology for explanations of the processes underlying how culture shapes mental health (Ryder et al., 2011).

Study 1 compared Euro-Canadians and Chinese migrants using the multiple mediation model. Independent selfconstrual was a significant mediator only for SIAS, not for OTKS. Consistent with previous research (e.g., Dinnel et al., 2002; Kleinknecht et al., 1997; Xie, Leong, \& Feng, 2008), Chinese migrants reported lower independent self-construal compared to Euro-Canadians, and through this effect of independent self-construal, Chinese migrants reported higher levels of SIAS. This is consistent with previous findings that people who were more concerned about autonomy from others were less likely to be socially anxious (e.g., Dinnel et al., 2002; Okazaki, 1997).

However, for OTKS, the two self-construals did not mediate the group differences we observed in Study 1. The influences of self-construals diminished when IU was in the model predicting OTKS. The results of Study 1 suggest that both "self-oriented" and the "other-oriented" fears are susceptible to IU, and the effect of IU is more salient among Chinese migrants than Euro-Canadians. In the multicultural Canadian social context, Chinese migrants may face many challenges during daily social interactions with people from different cultural backgrounds, and social norms and rules might be very different from what they have been used to in China. Levels of uncertainties during social interactions with others might be higher for Chinese migrants, compared to their Euro-Canadian counterparts. As a result, Chinese migrants might find social interactions with others very anxiety provoking and be fearful of offending others due to the novel and ambiguous situations they often face.

In order to ensure the cultural variations we observed in Study 1 are generalizable, we conducted a parallel study in three cultural contexts to further examine population specific variables that are related to and implicated in social anxiety and OTKS (Norenzayan \& Heine, 2005). Different indirect effects (mediations) in the three cultural group comparisons were found in Study 2. Unlike in Study 1, the indirect effect of IU was not statistically significant for SIAS or OTKS in the comparison between Euro-Canadians and Chinese. This 
finding may be due to the significant mean differences in SIAS, $t(284)=-8.87, p<.001$, and OTKS, $t(245)=-17.69$, $p<.001$, between Euro-Canadians in Study 1 and Study 2 with participants from Study 2 rated higher on both scales. There were no significant differences in SIAS and OTKS for Chinese participants between the two studies. Therefore, the lack of a statistically significant indirect effect of IU in Study 2 may indeed be due to the higher mean levels of SIAS and OTKS for Euro-Canadian participants.

Another possible explanation is rooted in the local sociocultural contexts. A post hoc analysis indicated that Chinese migrants in Canada (Study 1) and Chinese participants in China (Study 2) had similar levels of IU, $t(196)=-.30$, $p=.76$. Our results suggest that the effect of IU found in Study 1 is not because Chinese migrants are less tolerant of uncertainty than the Chinese in Study 2. Instead, the result indicates that not only ethnocultural backgrounds but also the specific local socio-cultural contexts where participants live (e.g., Chinese migrants in a multicultural Canadian context) influence levels of social anxiety and OTKS. Specific local socio-cultural contexts have their own social norms and daily practices reflecting different ecologies and histories; these local contexts also have their own unique cultural meanings of how to be and how to be well (Plaut, Markus, Treadway, \& Fu, 2012). What is deemed to be socially acceptable and desired varies from one place to another. Socialization processes in a given cultural context can certainly reflect people's psychological processes. For instance, independent self-construal is often found to be related to less social anxiety (e.g., Dinnel et al., 2002; Hong \& Woody, 2007; Okazaki et al., 2002). However, the local socio-cultural context where these psychological processes become salient or even pathological is also important because both cultural contexts and the self are constantly undergoing negotiation and reformation within these local socio-cultural contexts (Kashima et al., 2011). As a result, it is crucial to consider cultural variations in social anxiety, OTKS, and psychopathology in general as a process in a given context, not a static mean difference between groups across all situations and locations.

In other cultural contrasts, IU appears to be a much more important factor contributing to the variations between Euro-Canadian and Japanese contexts than between EuroCanadian and Chinese contexts on both SA and OTKS. There were several significant mediation results involving comparisons of the Chinese and Japanese groups, comparisons that are rarely made in the literature. Interdependent selfconstrual was not a significant mediator for SIAS in this comparison, but it was a significant mediator for OTKS. This was consistent with our conceptualization of OTKS as a form of anxiety that is more "other-oriented" than the traditional "Western" defined social anxiety, but this was not the case in the Euro-Canadian vs. Japanese comparison.

In addition, Japanese participants reported lower levels of interdependent self-construal than did Chinese participants, and the lower levels of interdependent self-construal positively related to higher levels of OTKS. Since the comparison between Japanese and Chinese is rarely made, it is unclear why Japanese participants would rate lower in interdependent self-construal compared with the Chinese participants. Our results provide support for Hofstede (2001) and Hofstede et al. (2010) that at the country level, Japanese tend to be more individualistic in Asian standards. Moreover, cultural differences in self-construals have often yielded mixed results, especially for interdependent self-construal (Kashima et al., 2011; Kleinknecht et al., 1997; Matsumoto, 2002; Xie et al., 2008). This may be due to the self-report measures we used, which only reflects the attitudinal aspects of selfconstruals (Norasakkunkit et al., 2012) and fails to fully capture the dynamic mutual constitution between culture and the self (Kashima et al., 2011; Markus \& Kitayama, 2010).

Japanese participants were also more likely to report higher levels of IU, in comparison to Chinese participants. IU in turn was associated with higher levels of both social anxiety and OTKS. Our results indirectly support Heinrichs and colleagues' (2006) hypothesis that social norms are related to social anxiety, and the anxiety-provoking sanctions of inappropriate social behaviours may be greater for cultural groups with stringent social norms than cultural groups favoring more relaxed social norms. Moment-to-moment social interactions with others are inherently complex and potentially unpredictable; consequently, heightened levels of IU becomes a particularly salient factor in eliciting social anxiety and OTKS.

The centrality of IU is particularly salient in Japanese cultural contexts, where appropriate behaviours are embedded in elaborate rules of daily social interactions - ranging from the degrees of bowing to the manner of pouring alcoholic beverages (e.g., Hendry, 2003; Nakane, 1970; Sugimoto, 2009). There is much opportunity, therefore, to be negatively evaluated. Even though the degrees to which Japan and China differ in terms of social norms was not directly tested, the results of Study 2 provide evidence that there are different pathways associated with SA and OTKS in the Japanese vs. Chinese cultural context comparisons. The influence of IU on eliciting SA and OTKS was stronger in the Japanese cultural context, as predicted, than in the Euro-Canadian and Chinese cultural contexts. Future studies should go beyond the comparison between the "West" and the "East", and focus more on specific cultural context comparisons, such as Japanese vs. Chinese or Japanese vs. Koreans to replicate the results obtained here.

Several limitations should be kept in mind while interpreting the outcomes of the two studies presented above. Participants in these two studies consisted mostly of university students. Future studies should extend this approach to community and clinical samples for replication. The two stud- 
ies were all based on self-report measures. Experimental manipulation of uncertainty or intolerance of uncertainty as well as observational measures are necessary to investigate the causal influence of IU on OTKS and social anxiety in multiple cross-cultural contexts. Furthermore, even though we have used the exact same items in each scale in Study 2 in order to ensure comparability between Study 1 and 2, Differential Item Functioning (DIF) analysis showed that certain items in IUS, SCS and OTKSQ ${ }^{3}$ in Study 2 displayed uniform DIF. This may be due to different languages used in Study 2 and it also may be due to some items being understood differently in different cultural contexts and therefore be responded differently. Future investigations perhaps should focus on refining certain items in these three scales. Furthermore, as mentioned earlier, the two samples of EuroCanadian participants in Study 1 and Study 2 had significantly different means for SIAS and OTKS. The interpretation of Euro-Canadian vs. Chinese group comparison should therefore be made cautiously. Future studies should be conducted using similar models and more refined items in each scale in order to replicate our results.

Finally, different significant mediators for predicting SIAS and OTKS in the four cultural contexts (EuroCanadians, Chinese migrants in Canada, Chinese and Japanese) have shed light on the importance of comparing multiple pathways (i.e., IU and self-construals) simultaneously leading to social anxiety and OTKS. This study also helps to give credence to IU in further unpacking its similar influences on SA and OTKS. On the one hand, the two studies suggest psychological universality of the effect of IU on SA and OTKS, such that IU was positively related to both SA and OTKS across all cultural groups (see Tables 2 and 3). On the other hand, the extent to which IU is contributing to the cultural variations in SA and OTKS depends on the specific group comparisons. This pattern also holds true for the effects of independent vs. interdependent self-construals. In sum, the evidence obtained in this paper points to both psychological universality and cultural variability for aspects of SA and OTKS.

\section{References}

Alon, I. (2003). Chinese culture, organizational behavior, and international business management. Westport, CT: Praeger.

American Psychiatric Association. (2013). Diagnostic and statistical manual of mental disorders, fifth edition (DSM-5). American Psychiatric Publishing.

Boelen, P. A., \& Reijntjes, A. (2009). Intolerance of uncertainty and social anxiety. Journal of Anxiety Disorders, 23 (1), 130-135. doi: 10.1016/j.janxdis.2008.04.007

Buhr, K., \& Dugas, M. (2002). The intolerance of uncertainty scale: Psychometric properties of the English version. Behaviour Research and Therapy, 40 (8), 931-946. doi: 10.1016/S0005-7967(01)00092-4

Carleton, R. N., Collimore, K. C., \& Asmundson, G. J. (2010). "It's not just the judgements-it's that I don't know": Intolerance of uncertainty as a predictor of social anxiety. Journal of Anxiety Disorders, 24(2), 189-195. doi: 10.1016/j.janxdis.2009.10.007

Carleton, R. N., Mulvogue, M. K., Thibodeau, M. A., McCabe, R. E., Antony, M. M., \& Asmundson, G. J. (2012). Increasingly certain about uncertainty: Intolerance of uncertainty across anxiety and depression. Journal of Anxiety Disorders, 26 (3), 468-479. doi: 10.1016/j.janxdis.2012.01.011

Choy, Y., Schneier, F. R., Heimberg, R. G., Oh, K.-S., \& Liebowitz, M. R. (2008). Features of the offensive subtype of Taijin-Kyofu-Sho in US and Korean patients with DSMIV social anxiety disorder. Depression and Anxiety, 25(3), 230-240. doi: 10.1002/da.20295

Clarvit, S. R., Schneier, F. R., \& Liebowitz, M. R. (1996). The offensive subtype of taijin-kyofu-sho in New York City: The phenomenology and treatment of a social anxiety disorder. Journal of Clinical Psychiatry, 57 , 523-527. doi: 10.4088/JCP.v57n1104

Dinnel, D., Kleinknecht, R., \& Tanaka-Matsumi, J. (2002). A cross-cultural comparison of

social phobia symptoms. Journal of Psychopathology and Behavioral Assessment, 24 (2), 75-84. doi: 10.1023/A:1015316223631

Draguns, J. G., \& Tanaka-Matsumi, J. (2003). Assessment of psychopathology across and within cultures: Issues and findings. Behaviour Research and Therapy, 41 (7), 755-776. doi: 10.1016/S0005-7967(02)00190-0

Dugas, M. J., Schwartz, A., \& Francis, K. (2004). Intolerance of uncertainty, worry, and depression. Cognitive Therapy and Research, 28 (6), 835-842. doi: 10.1007/s10608004-0669-0

Good, B., \& Kleinman, A. (1985). Culture and anxiety: Cross-cultural evidence for the patterning of anxiety disorders. In A. H. Tuma \& J. D. Maser (Eds.), Anxiety and the anxiety disorders (p. 297-323). Hillsdale, NJ, England: Lawrence Erlbaum Associates, Inc.

Harkness, J. A. (2003). Questionnaire translation. In J. A. Harkness, F. van de Vijver, \& P. P. Mohler (Eds.), Crosscultural survey methods (p. 35-56). Hoboken, NJ: John Wiley \& Sons.

Hayes, A. F. (2013). Introduction to mediation, moderation, and conditional process analysis: A regression-based approach (T. D. Little, Ed.). Guilford Press: New York.

Hayes, A. F., \& Cai, L. (2007). Using heteroskedasticityconsistent standard error estimators in OLS regression: An

\footnotetext{
${ }^{3}$ Items displayed DIF in IUS -2, 5, 9,21 (Buhr \& Dugas, 2002), Independent self-construal - 9, 24, 25, Interdependent selfconstrual - 4, 16, 17, 19 (Singelis, 1994), TKSQ -30 (Choy et al., 2008).
} 
introduction and software implementation. Behavior Research Methods, 39 , 709-722. doi: 10.3758/BF03192961

Hayes, A. F., \& Preacher, K. J. (in press). Statistical mediation analysis with a multicategorical independent variable. British Journal of Mathematical and Statistical Psychology. doi: 10.1111/bmsp.12028

Heinrichs, N., Rapee, R. M., Alden, L. A., Bögels, S., Hofmann, S. G., Oh, K. J., \& Sakano, Y. (2006). Cultural differences in perceived social norms and social anxiety. $B e$ haviour Research and Therapy, 44 (8), 1187-1197. doi: 10.1016/j.brat.2005.09.006

Hendry, J. (2003). Understanding Japanese society. London and New York : Routledge.

Hofmann, S. G., Asnaani, A., \& Hinton, D. E. (2010). Cultural aspects in social anxiety and social anxiety disorder. Depression $\mathcal{E}$ Anxiety, 27 (12), 1117-1127. doi: 10.1002/da.20759

Hofstede, G. (1984). Culture's consequences: International differences in work-related values. Beverly Hills, CA: Sage.

Hofstede, G. (2001). Culture's consequences: Comparing values, behaviors, institutions, and organizations across nations (2nd ed.). Beverly Hills, CA: Sage.

Hofstede, G., Hofstede, G. J., \& Minkov, M. (2010). Cultures and organizations: Software of the mind (3rd ed.). New York: McGraw-Hill USA. Retrieved from http://geerthofstede.com/japan.html

Hong, J. J., \& Woody, S. R. (2007). Cultural mediators of self-reported social anxiety. Behaviour Research And Therapy, 45 (8), 1779-1789. doi: 10.1016/j.brat.2007.01.011

Iwase, M., Nakao, K., Takaishi, J., Yorifuji, K., Ikezawa, K., \& Takeda, M. (2000). An empirical classification of social anxiety: Performance, interpersonal and offensive. Psychiatry and Clinical Neurosciences, 54 (1), 67-76. doi: 10.1046/j.1440-1819.2000.00639.x

Kasahara, Y. (1974). Fear of eye-to-eye confrontation among neurotic patients in Japan. In T. S. Lebra \& W. P. Lebra (Eds.), Japanese culture and behaviour (p. 396-406). Honolulu: University of Hawaii Press.

Kasahara, Y. (1987). Social phobia in Japan. In Proceedings of the first cultural psychiatry symposium between Japan and Korea in Seoul, Korea. doi: 10.1177/136346158802500213

Kashima, Y., Koval, P., \& Kashima, E. S. (2011). Reconsidering culture and self. Psychological Studies, 56 (1), 12-22. doi: 10.1007/s12646-011-0071-4

Kim, J., Rapee, R. M., \& Gaston, J. E. (2008). Symptoms of offensive type taijin-kyofusho among Australian social phobics. Depression And Anxiety, 25 (7), 601-608. Retrieved from http://www.ncbi.nlm.nih.gov/pubmed/17607747

Kirmayer, L. (1991). The place of culture in psychiatric nosology: Taijin kyofusho and DSM-III-R. Jour- nal of Nervous and Mental Disease, 179, 19-28. doi: 10.1097/00005053-199101000-00005

Kleinknecht, R., Dinnel, D., Kleinknecht, E., Hiruma, N., \& Harada, N. (1997). Cultural factors in social anxiety: A comparison of social phobia symptoms and taijin kyofusho. Journal of Anxiety Disorders, 11 (2), 157-177. doi: 10.1016/S0887-6185(97)00004-2

Lin, T. (2013). The concepts of "politeness": A comparative study in Chinese and Japanese verbal communication. Intercultural Communication Studies, 22 (2), 151-165.

Louie, K. (2008). The Cambridge companion to modern Chinese culture. Cambridge; New York: Cambridge University Press.

Markus, H. R., \& Kitayama, S. (2010). Cultures and selves: A cycle of mutual constitution. Perspectives on Psychological Science, 5 (4), 420-430. doi: $10.1177 / 1745691610375557$

Matsumoto, D. (2002). The new Japan: Debunking seven cultural stereotypes. Yarmouth, Me: Intercultural Press.

Mattick, R. P., \& Clarke, J. C. (1998). Development and validation of measures of social phobia scrutiny fear and social interaction anxiety. Behaviour Research and Therapy, 36 , 455-470. doi: 10.1016/S0005-7967(97)10031-6

McEvoy, P. M., \& Mahoney, A. E. J. (2012). To be sure, to be sure: Intolerance of uncertainty mediates symptoms of various anxiety disorders and depression. Behavior Therapy, 43 (3), 533-545. doi: 10.1016/j.beth.2011.02.007

McKnight, P. E., McKnight, K. M., Sidani, S. F., \& José, A. (2007). Missing data: A gentle introduction (D. A. Kenny, Ed.). New York, NY, US: Guilford Press.

Nagata, T., van Vliet, I., Yamada, H., Kataoka, K., Iketani, T., \& Kiriike, N. (2006). An open trial of paroxetine for the "Offensive Subtype" of Taijin Kyofusho and social anxiety disorder. Depression and Anxiety, 23 (3), 168-174. doi: 10.1002/da.20153

Nakane, C. (1970). Japanese society. Berkely, California: University of California Press. Norasakkunkit, V., Kitayama, S., \& Uchida, Y. (2012). Social anxiety and holistic cognition: Self-focused social anxiety in the United States and other-focused social anxiety in Japan. Journal of Cross-Cultural Psychology, 43 (5), 742-757. doi: 10.1177/0022022111405658

Norenzayan, A., \& Heine, S. J. (2005). Psychological universals: What are they and how can we know? Psychological Bullentin, 131 (5), 763-784. doi: 0.1037/00332909.131.5.763

Okazaki, S. (1997). Sources of ethnic differences between Asian American and White American college students on measures of depression and social anxiety. Journal of $A b$ normal Psychology, 106 (1), 52-60. doi: 10.1037/0021843X.106.1.52

Okazaki, S., Liu, J. F., Longworth, S. L., \& Minn, J. Y. (2002). Asian American-White American differences in 
expressions of social anxiety: A replication and extension. Cultural Diversity and Ethnic Minority Psychology, 8 (3), 234-247. doi: 10.1037/1099-9809.8.3.234

Ono, Y., Yoshimura, K., Yamauchi, K., Asai, M., Young, J., Fujuhara, S., \& Kitamura, T. (2001). Taijin kyofusho in a Japanese community population. Transcultural Psychiatry, 38 (4), 506-514. doi: 10.1177/136346150103800408

Plaut, V. C., Markus, H. R., Treadway, J. R., \& Fu, A. S. (2012). The cultural construction of self and well-being: A tale of two cities. Personality and Social Psychology Bulletin, 38 (12), 1644-1658. doi: 10.1177/0146167212458125

Rector, N. A., Kocovski, N. L., \& Ryder, A. G. (2006). Social anxiety and the fear of causing discomfort to others: Conceptualization and treatment. Journal of Social and Clinical Psychology, 25 (8), 906-918. doi: 10.1007/s10608-0069050-9

Ryder, A. G., Ban, L. M., \& Chentsova-Dutton, Y. E. (2011). Towards a cultural-clinical psychology. Social and Personality Psychology Compass, 5, 960-975. doi: 10.1111/j.1751-9004.2011.00404.x

Singelis, T. M. (1994). The measurement of independent and interdependent self-construals. Personality and Social Psychology Bulletin, 20 (5), 580-591. doi: 10.1177/0146167294205014

Sugimoto, Y. (2009). The Cambridge companion to modern Japanese culture. Cambridge, UK: Cambridge University Press.

Takahashi, T. (1989). Social phobia syndrome in Japan. Comprehensive Psychiatry, 30 (1), 45-52. doi: 10.1016/0010-440X(89)90117-X

Xie, D., Leong, F. T. L., \& Feng, S. (2008). Culturespecific personality correlates of anxiety among Chinese and Caucasian college students. Asian Journal of Social Psychology, 11 , 163-174. doi: 10.1111/j.1467-839X.2008.00253.x

\section{Appendix}


Table 1

Internal Consistency Across All Scales - Cronbach's Alpha in Study 1.

\begin{tabular}{lccccccc}
\hline Scale & Items & \multicolumn{2}{c}{$\begin{array}{c}\text { Euro-Canadians } \\
(n=267)\end{array}$} & \multicolumn{2}{l}{$\begin{array}{l}\text { Chinese } \\
(n=109)\end{array}$} \\
\hline Ind SC & 15 & .71 & $(5.06$, & $.62)$ & .77 & $(4.69$, & $.67)$ \\
Inter SC & 15 & .72 & $(4.54$, & $.63)$ & .82 & $(4.74$, & $.68)$ \\
IUS & 27 & .91 & $(1.67$, & $.46)$ & .95 & $(2.39$, & $.75)$ \\
SIAS & 20 & .87 & $(.80$, & $.48)$ & .90 & $(1.35$, & $.61)$ \\
OTKSQ & 20 & .89 & $(1.26$, & $.29)$ & .94 & $(1.98$, & $.60)$ \\
\hline
\end{tabular}

Note. Means and standard deviations are in parentheses. Ind SC is Independent Self-Construal, Inter SC is Interdependent Self-Construal, IUS is Intolerance of Uncertainty Scale, SIAS is Social Interaction Anxiety Scale, OTKSQ is Offensive-type Taijin Kyofusho Questionnaire.

\section{Table 2}

Correlations Among Variables in Study 1.

\begin{tabular}{lccccc}
\hline Variables & 1 & 2 & 3 & 4 & 5 \\
\hline 1. Independent Self-Construal & - & .10 & $-.15^{*}$ & $-.32^{* *}$ & -.07 \\
2. Interdependent Self-Construal & $.50^{* *}$ & - & $.13^{*}$ & $.16^{* *}$ & $.20^{* *}$ \\
3. Intolerance of Uncertainty Scale & -.05 & .13 & - & $.43^{* *}$ & $.38^{* *}$ \\
4. Social Interaction Anxiety Scale & $-.37^{* *}$ & $-.23^{* *}$ & $.52^{* *}$ & - & $.35^{* *}$ \\
5. Offensive-Type TKS Questionnaire & -.08 & .04 & $.27 * *$ & $.35^{* *}$ & - \\
\hline
\end{tabular}

Note. ${ }^{*} p<.05, * * p<.01$. Upper diagonal contains coefficients for Euro-Canadian participants, and lower diagonal for Chinese migrants. 
Table 3

Correlations Among Variables in Study 2.

\begin{tabular}{lllllll}
\hline & 1 & 2 & 3 & 4 & 5 \\
\hline
\end{tabular}

Euro-Canadians

1. Independent Self-Construal $\quad-\quad .09 \quad-.27 * * \quad-.52 * * \quad-.35 * *$

2. Interdependent Self-Construal $\quad-\quad .24 * * \quad .19^{* *} \quad .25^{* *}$

3. Intolerance of Uncertainty Scale $\quad-\quad .63 * * \quad .48^{* *}$

4. Social Interaction Anxiety Scale $\quad-\quad .49^{* *}$

5. Offensive-Type TKS Questionnaire

\section{Chinese}

1. Independent Self-Construal $\quad-\quad \begin{array}{lllll}.69 * * & -.03 & -.14 & -.05\end{array}$

2. Interdependent Self-Construal $\quad-\quad-\quad \begin{array}{lllll}-.05 & -.10 & .15\end{array}$

3. Intolerance of Uncertainty Scale $\quad-\quad \begin{array}{lll}.33 * * & .16\end{array}$

4. Social Interaction Anxiety Scale $\quad-\quad .32^{* *}$

5. Offensive-Type TKS Questionnaire

Japanese

1. Independent Self-Construal $\quad-\quad \begin{array}{lllll}.10 & -.10 & -.37 * * & -.11\end{array}$

2. Interdependent Self-Construal $\quad-\quad \begin{array}{llll}.23 * * & .21 * * & .34 * *\end{array}$

3. Intolerance of Uncertainty Scale $\quad-\quad .48^{* *} \quad .28^{* *}$

4. Social Interaction Anxiety Scale $\quad-\quad .46^{* *}$

5. Offensive-Type TKS Questionnaire 
Table 4

Internal Consistency Across All Scales-Cronbach's Alpha in Study 2.

\begin{tabular}{lccccccccccc}
\hline Scale & Items & \multicolumn{2}{c}{$\begin{array}{c}\text { Euro-Canadians } \\
(n=198)\end{array}$} & \multicolumn{2}{c}{$\begin{array}{c}\text { Chinese } \\
(n=127)\end{array}$} & \multicolumn{3}{c}{ Japanese } \\
& & \multicolumn{3}{c}{$(n=124)$} \\
\hline Ind SC & 15 & .77 & $(4.83$, & $.68)$ & .80 & $(4.61$, & $.76)$ & .73 & $(4.54$, & $.69)$ \\
Inter SC & 15 & .68 & $(4.69$, & $.59)$ & .83 & $(5.03$, & $.73)$ & .71 & $(4.58$, & $.64)$ \\
IUS & 27 & .96 & $(2.27$, & $.84)$ & .88 & $(2.37$, & $.55)$ & .93 & $(2.91$, & $.72)$ \\
SIAS & 20 & .95 & $(1.41$, & $.87)$ & .88 & $(1.21$, & $.52)$ & .90 & $(1.79$, & $.74)$ \\
OTKSQ & 20 & .93 & $(2.07$, & $.63)$ & .90 & $(2.17$, & $.50)$ & .94 & $(2.30$, & $.70)$ \\
\hline
\end{tabular}

Note. Means and standard deviations are in parentheses. Ind SC is Independent Self-Construal, Inter SC is Interdependent Self-Construal, IUS is Intolerance of Uncertainty Scale, SIAS is Social Interaction Anxiety Scale, OTKSQ is Offensive-type Taijin Kyofusho Questionnaire. 


\section{Figure 1}

Mediation Models in Study 1 and 2. *Only Independent Self-construal and Intolerance of Uncertainty were entered in Study 1 for SIAS. **Only Interdependent Self-construal and Intolerance of Uncertainty were entered in Study 2 for SIAS.

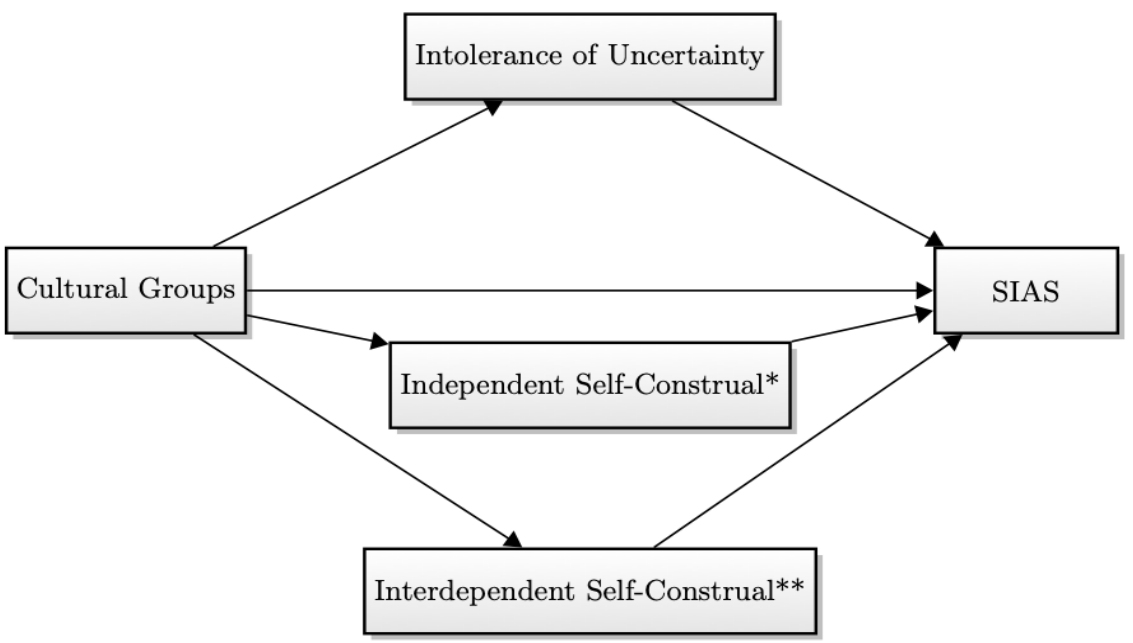

(a) Panel A: Multiple Mediation Model for SIAS

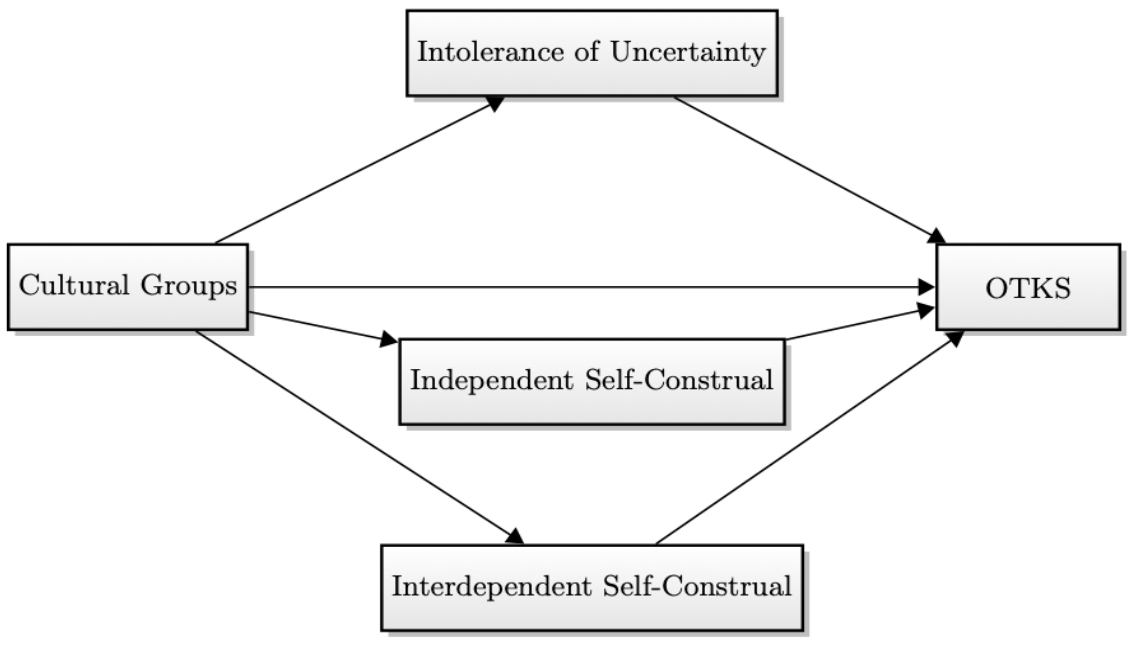

(b) Panel B: Multiple Mediation Model for OTKS 